\title{
Aplicación de tecnologías de teledetección al estudio de biomasa forestal
}

\author{
Luis Fernando Sánchez Sastre ${ }^{1}$, José-Luis Marcos-Robles², Eliecer Herrero Llorente', \\ Salvador Hernández Navarro ${ }^{1}$, Paula Carrión Prieto ${ }^{1}$ \\ lux@iaf.uva.es, jlmarcos@iaf.uva.es, eliecer@iaf.uva.es, inpaisal@iaf.uva.es, \\ paula.carrion@alumnos.uva.es \\ ${ }^{1}$ Departamento de Ingeniería Agrícola y Forestal, E.T.S.II.AA., Universidad de Valladolid, Campus La Yutera - \\ Av Madrid 57, 34005 Palencia, España. \\ ${ }^{2}$ Departamento de Ciencia de los Materiales, E.T.S.I.I.A.A., Universidad de Valladolid, Campus La Yutera - \\ Av Madrid 57, 34005 Palencia, España.
}

DOI: $10.17013 /$ risti.19.61-76

Resumen: Este estudio tiene como objetivo conocer el estado inicial de la vegetación de una zona forestal del Prepirineo, así como generar los datos necesarios para estimar la cantidad de biomasa, y por lo tanto de carbono capturado en la misma, al inicio del proyecto europeo LIFE+ Operación $\mathrm{CO}_{2}$. Para ello se han utilizado tecnologías de teledetección como procesamiento de datos LiDAR, imágenes por satélite, fotogrametría de rango cercano mediante RPAs o drones. Todo ello junto con trabajo de apoyo sobre el terreno y simulaciones con modelos matemáticos de crecimiento. Los resultados muestran la gran utilidad de estas tecnologías para la obtención de información geolocalizada sobre todo en áreas extensas y de orografía montañosa. Además se constata la validez y fiabilidad de estos métodos por lo que resultan adecuados para el seguimiento y evaluación de actividades agroforestales.

Palabras-clave: LiDAR; teledetección; drones; biomasa; carbono.

\section{Remote sensing technologies applied to forestry biomass studies.}

\begin{abstract}
This study aims to evaluate the initial conditions of the vegetation of a pre-Pyrinees forestry area, as well as to generate data for estimating biomass and hence uptaken carbon, at the beginning of the European project LIFE+ Operation $\mathrm{CO}_{2}$. In order to achieve this, remote sensing technologies such as LiDAR data processing, satellite images, close-range photogrammetry by using RPA (drones) were used. Besides, support fieldwork and simulations with a growth model were conducted. Results show the great utility of these technologies to obtain georeferred information, especially, in large and mountainous areas. In addition, reliability and validity of these methodologies were demonstrated and therefore they are suitable for monitoring and evaluating purposes in agroforestry activities.
\end{abstract}

Keywords: LiDAR; remote-sensing; drones; biomass; carbon. 


\section{Introducción}

Los ecosistemas forestales juegan un papel clave en el ciclo global del carbono debido al intercambio natural de carbono entre bosques y atmósfera a través de la fotosíntesis, respiración, descomposición y combustión (IPPC, 2000). La biomasa, al acumular carbono, actúa como sumidero de $\mathrm{CO} 2$ atmosférico secuestrando anualmente entre 0.01 y $1.08 \mathrm{t} \mathrm{C} \mathrm{ha}^{-1}$ en la región mediterránea (Croitoru \& Merlo, 2005). En España, aproximadamente el $19 \%$ del total de las emisiones anuales son capturadas por los bosques (Montero, Ruiz-Peinado \& Muñoz, 2005).

El presente trabajo se desarrolla dentro del marco del proyecto LIFE11 ENV/ES/535 Operation $\mathrm{CO}_{2}$ (http://operacionco2.es), el cual tiene como objetivo general demostrar la viabilidad económica de proyectos agroforestales de secuestro de carbono en Europa mediante la conservación y manejo de bosques y las prácticas agroforestales integradas (Sánchez Sastre et al., 2016). Es por ello que conocer el estado inicial de las zonas de estudio y estimar la biomasa presente son acciones fundamentales para establecer una línea base al inicio del proyecto. De esta manera, se obtienen unos valores de referencia con los que poder comparar la situación al final del proyecto, en el que se espera un incremento en la captura de $\mathrm{CO} 2$ mediante las acciones desarrolladas a lo largo de los años de funcionamiento del mismo.

La mayoría de países no poseen un inventario de datos expresados directamente en términos de biomasa y usan en su lugar factores de conversión y/o expansión aplicados a los datos de crecimiento de existencias para estimar la biomasa (Marklund \& Schoane, 2006). Por lo tanto se requieren métodos para proporcionar estimaciones precisas de biomasa forestal y sus cambios para incrementar nuestro conocimiento del papel de los bosques en el ciclo del carbono.

Así mismo, los métodos convencionales de estimación de biomasa están basados en medidas de campo que a pesar de ser métodos más directos están generalmentelimitados en términos espaciales y temporales a la hora de efectuar muestreos. Zonas extensas, agrestes o de orografía montañosa complican aún más este tipo de trabajo. Sin embargo, el uso de sensores remotos, independientemente de la plataforma utilizada: satélites, aviones o RPAs (Aeronaves Pilotadas Remotamente, comúnmente conocidas como drones), proporcionan un método para generar detallada información, explícita espacialmente, sobre biomasa forestal dado su potencial para suministrar información en un amplio rango de escalas temporales y espaciales. No obstante, se necesitan desarrollar métodos apropiados para extraer datos relevantes (García, Riaño, Chuvieco \& Danson, 2010; Vázquez et al., 2016).

\section{Material y métodos}

Los objetivos perseguidos con este estudio han sido, por una parte, obtener los datos necesarios para una posterior estimación de la cantidad de biomasa existente en aquellas zonas de monte prepirenaico sobre las que trabaja el proyecto, y por otra, evaluar el estado actual de una de ellas y su posible evolución en los próximos años.

\section{1. Área de estudio}

De las 3 localizaciones del proyecto, este estudio se centra en un área forestal localizada en el término municipal de Figols i Alinyá (Lérida), sito en la comarca catalana del Alt 
Urgell, dentro del Espacio Natural Montaña de Alinyà (Figura 1) perteneciente a la Fundación Catalunya-La Pedrera (FCLP). Este espacio natural, con una altitud entre 500 y $2.380 \mathrm{~m}$ de altura y un relieve accidentado proporciona una gran diversidad de paisajes y ambientes, pudiendo encontrarse bosques, pastos, matorral, altas cumbres y accidentadas zonas de peñascos que permiten una gran riqueza faunística y botánica. Así, Alinyà es un famoso escenario y refugio de especies emblemáticas como el rebeco o el urogallo, y se observan con facilidad las cuatro especies de buitres que viven en Europa: buitre leonado, quebrantahuesos, buitre negro y alimoche.

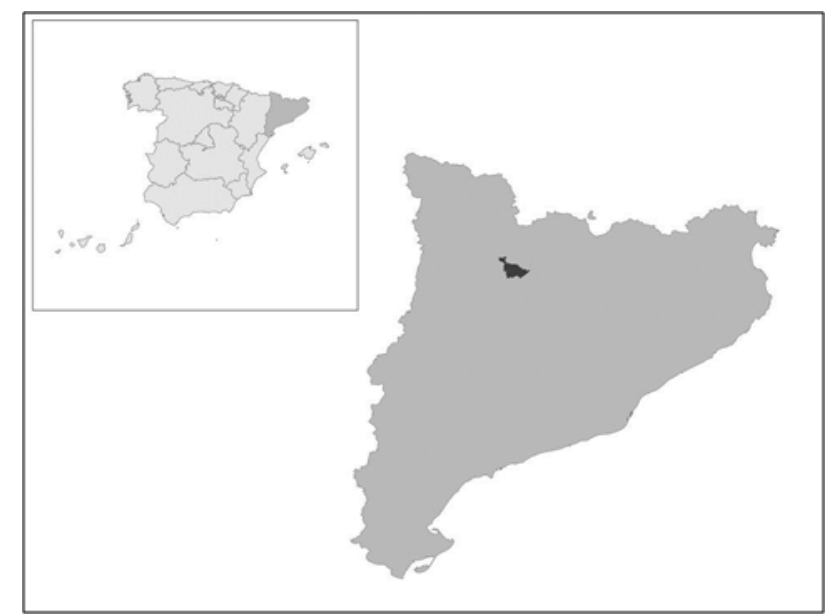

Figura 1 - Localización del municipio de Figols i Alinyà dentro de la comunidad autónoma de Cataluña, España

Dentro de dicho espacio natural se han seleccionado un total de 6,6 ha dedicadas a forestación, reforestación y restauración (acción ARR, Figura 2 izquierda) y 18 ha a la mejora de la gestión forestal (acción IFM, Figura 2 derecha).
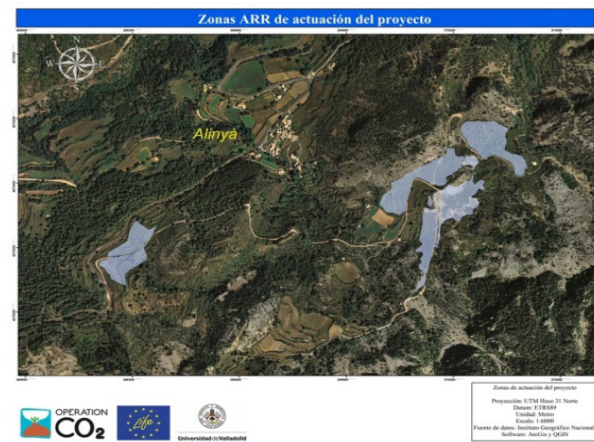

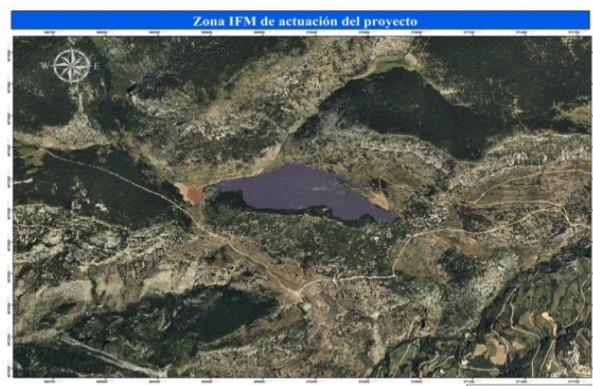

$\mathrm{CO}_{2}$

Figura 2 - Izquierda: localización de las 9 zonas dedicadas a la acción ARR. Derecha: zona donde se ejecuta la acción IFM 
Las zonas objetivo de actuación de la acción ARR son áreas degradadas donde la vegetación está compuesta mayoritariamente por formaciones de herbáceas, arbustivas y de matorral formadas por enebro (Juniperus oxycedrus), boj (Buxus sempervirens), espino blanco (Crataegus sp.), rosáceas y romero. En cuanto a las escasas formaciones de porte arbóreo hay que destacar el pino pudio (Pinus nigra), pino silvestre (Pinus sylvestris), rebollo (Quercus pyrenaica) y enebro (Juniperus oxycedrus). La zona dedicada a la acción IFM es una masa homogénea de Pinus sylvestris, con presencia de boj y espino, surgida por regeneración tras el abandono de las labores agrícolas durante el siglo XX.

A la hora de realizar la estimación de la biomasa presente en estas zonas, el alto grado de heterogeneidad, la dispersión de la mismas y las fuertes pendientes hacen que se dificulte enormemente el trabajo a pie de campo y la representatividad de los datos muestrales. Para solventar esta problemática se estableció una metodología apoyada en una combinación de tecnologías de teledetección remota: datos LiDAR (Light Detection and Ranging), imágenes de alta resolución y fotogrametría mediante RPA, SIGs (Sistemas de Información Geográfica), software estadístico, modelos de crecimiento. Todo ellos apoyado con datos de campo.

\subsection{Tecnología LiDAR.}

El LiDAR es un sistema activo de detección remota basada en un escáner láser, generalmente aerotransportado, que proporciona información tridimensional de las superficies que escanea mediante la emisión de pulsos láser (Figura 3) que permite medir distancias, y generar una nube de puntos de elevación muy precisa y especialmente densa. Esto se consigue con la combinación de tres tecnologías: un láser, un sistema de referencia inercial de alta precisión (IMU, Inertial Measurement Unit) y un sistema de posicionamiento global por satélite. La combinación de estos tres sistemas, montados en un único instrumento, permite obtener con gran rapidez y precisión Modelos Digitales del Terreno (Baltsavias, 2008).

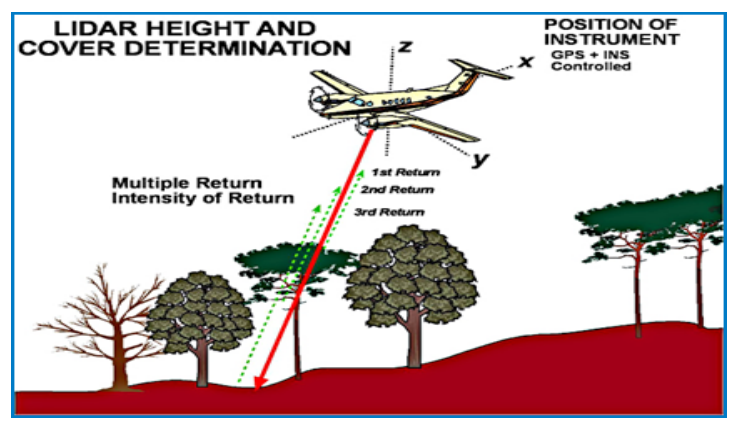

Figura 3 - Esquema de un vuelo LiDAR (Fuente: USDA - Departamento de Agricultura de Estados Unidos)

De esta forma, esta reciente tecnología presenta un gran potencial para muchos tipos de estudios y aplicaciones al proporcionar una alta cantidad de datos, así como la georeferenciación digital de la elevación de los mismos con gran precisión. 
Otra de las ventajas de esta nueva tecnología es que, al contrario que la imagen adquirida con cámaras fotogramétricas, los instrumentos LiDAR son sistemas de sensores activos y, como consecuencia, ofrece importantes ventajas sobre la fotogrametría, ya que puede penetrar a través de las copas de los árboles, facilitando información de la superficie que se encuentra bajo los mismos y permitiendo construir un modelo digital del terreno bajo la cubierta forestal (Renslow, Geenfield \& Guay, 2000). Las aplicaciones LiDAR más frecuentes en estudios forestales han sido para la determinación de atributos estructurales básicos, incluyendo la altura, la cobertura del dosel y los perfiles verticales, y partir de ellos medidas indirectas (Navarro-Cerrillo, Sánchez de la Orden, Gómez Bonilla, García Ferrer, Hernández Clemente \& Lanjeri, 2010).

El proceso que se ha seguido se puede resumir esquemáticamente en los siguientes pasos:

- Procesamiento de datos LiDAR mediante software específico

- Generación de MDTs (Modelos Digitales del Terreno): MDE (Modelo

- Digital de Elevaciones), MDS (Modelo Digital de Superficies) y MDHV (Modelo Digital de Altura de Vegetación)

- Cálculo de la FCC (Fracción Cabida Cubierta)

- Discriminación y determinación de estratos vegetativos

- Desarrollo de modelos numéricos para distintos parámetros

- Estimación del número de árboles según clases diamétricas

- Generación de información para su posterior utilización como datos de entrada de distintas ecuaciones de biomasa.

- Implementación de un SIG con los resultados obtenidos

La tecnología LiDAR todavía tiene alto coste económico pero, afortunadamente, en España se dispone de buenas fuentes de información geográfica (Porta et al., 2012). Concretamente, en este estudio, se han utilizado los datos provenientes de vuelos LiDAR realizados por el IGN (Instituto Geográfico Nacional) dentro del plan PNOA (Plan Nacional de Ortografía Aérea).

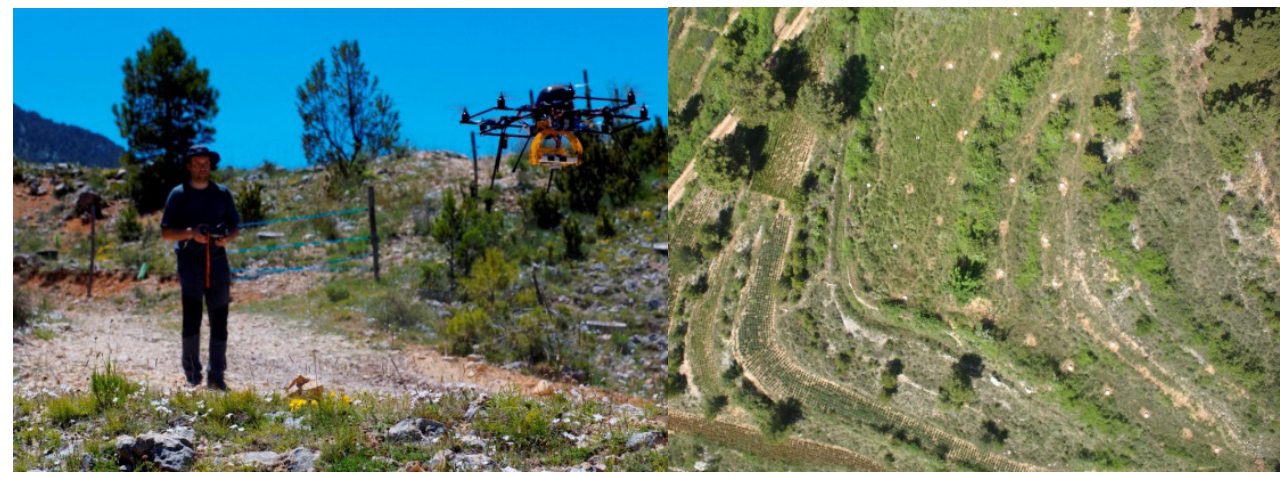

Figura 4 - Izquierda: RPA de 8 rotores con cámara de visible durante maniobra de despegue. Derecha: ejemplo de fotografía capturada desde el RPA. 


\subsection{Fotogrametría mediante RPA}

Asimismo se realizaron vuelos con un RPA tipo multi-rotor (Figura 4 izquierda) equipado con cámara digital RGB (espectro visible) de alta resolución que genera una cobertura fotogramétrica de alta resolución espacial (Figura 4 derecha) mediante un adecuado solapamiento entre fotogramas y entre pasadas. Los resultados cartográficos así obtenidos han sido ortofotos de alta resolución, modelos MDS (Modelo Digital de Superficies) y MDE (Modelo de Elevaciones) de precisión, generados a partir de una nube de puntos $3 \mathrm{D}$ determinados fotogramétricamente.

\subsection{Teledetección mediante imágenes satélite.}

Para el estudio de la evolución de la masa arbórea de la zona IFM en los últimos años, fueron adquiridas 7 imágenes TM (Thematic Mapper), del satélite Landsat 5 correspondientes a la escena con path 198 y row 31. Estas imágenes comprenden parte del territorio de Cataluña incluyendo el Espacio Natural de Alinyà y fueron captadas en las fechas de 28 de julio de 1984, 01 de julio de 2003, 06 de junio de 2006, 28 de junio de 2008, 01 de julio de 2009, 04 de julio de 2007 y 21 de junio de 2006. A partir de dichas imágenes, tras el procesamiento de los datos matriciales se calcularon los índices de vegetación NDVI (Índice de Vegetación por Diferencia Normalizada) propuesto por Rouse, Haas, Schell \& Deering (1973) y SAVI (Índice de Vegetación Ajustado al Suelo) propuesto por Huete (1988).

\subsection{Trabajo de campo}

Como soporte a las distintas metodologías de teledetección utilizadas en este trabajo, la información obtenida ha sido ha sido complementada y contrastada con los datos recogidos en distintas visitas a la zona. Este trabajo de campo ha sido de diversa naturaleza como por ejemplo, identificación de especies, estimación de la edad de los árboles (ver Figura 5 izquierda), medición de distintos parámetros dendrométricos (ver Figura 5 derecha). La utilización de la teledetección permite reducir todo este trabajo a actuaciones puntuales de apoyo y verificación. Como se puede apreciar en la Figura 5
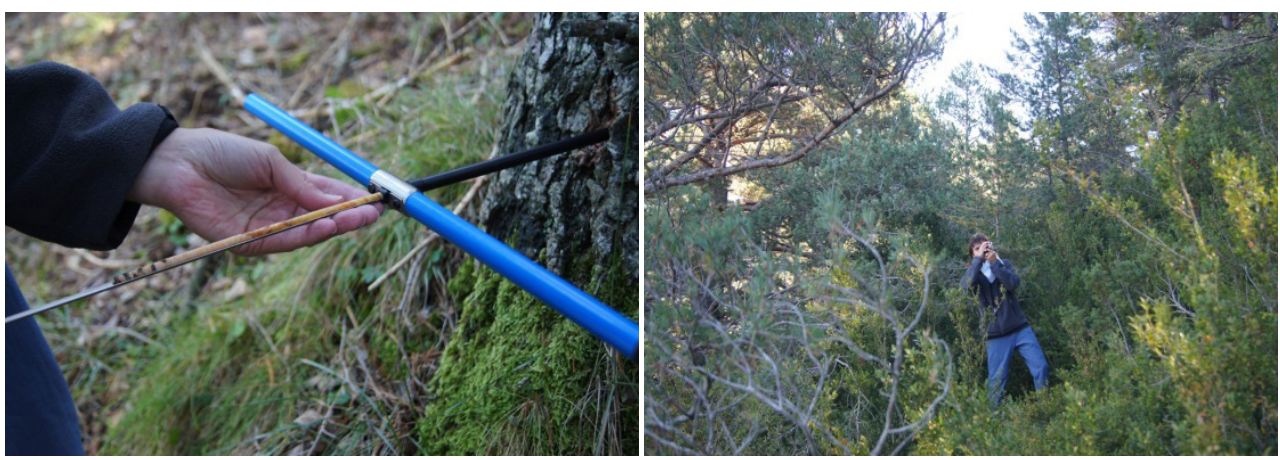

Figura 5 - Izquierda: extracción de un "core" de Pinus sylvestris de la zona IFM para la determinación de su edad. Derecha: medición de altura con hipsómetro de un pie de Pinus sylvestris en la zona IFM. 
derecha, zonas en pendiente y con una alta densidad de vegetación dificultarían enormemente la realización de un inventario clásico.

\subsection{Modelo de simulación}

A la hora de predecir el comportamiento futuro de la masa de Pinus sylvestris de la zona IFM se ha recurrido al modelo de simulación CO2FIX v3.1 (Schelhaas, van Esch, Groen, de Jong, Kanninen, Liski, et al., 2004) desarrollado dentro del proyecto europeo CASFOR (Secuestro de Carbono en Forestación y Gestión Forestal Sostenible). Para completar los datos que reclama como entrada el modelo se realizó un estudio climático de la zona, se recurrió a tablas de producción y se recabaron datos a pie de campo.

Se realizaron diversas simulaciones tanto para un modelo de gestión sin ninguna actuación (dejando al bosque en su evolución actual), como para el modelo de gestión propuesto por el CREAF (Centro de Investigación Ecológica y Aplicaciones Forestales) denominado Pso5 (Piqué, Beltrán, Vericat, Cervera, Farriol \& Baiges, 2011) desarrollado en el marco del proyecto LIFE12 ENV/ES/o00730 Demorgest y enfocado a una mayor captura de $\mathrm{CO}_{2}$.

\subsection{Resumen del software empleado}

Para el visionado de la nube de puntos LiDAR como ficheros en formato “.las" se ha utilizado el software gratuito FugroViever ${ }^{\mathrm{TM}}$, perteneciente a la compañía holandesa Fugro.

Para el análisis, filtrado, cálculo de variables y visionado de puntos se ha recurrido al software de libre distribución FUSION/LDV desarrollado por el Forest Service USDA (Servicio Forestal del Departamento de Agricultura de los Estados Unidos). Complementariamente para la gestión y el filtrado se utilizó el software Lastools (Isenburg, 2008).

En cuanto a software SIG se ha trabajado, por una parte, con el programa de código libre y gratuito QuantumGIS desarrollado por la fundación OSGeo (Open Source Geospatial Foundation), y por otra, para el cálculo de índices y la generación de los mapas de resultados se ha utilizado el programa $\operatorname{ArcMap}^{\mathrm{TM}} 10$ (ArcGIS $\left.{ }^{\circledR}\right)$ bajo licencia de Esri.

El procesado de las imágenes obtenidas con las cámaras fotográficas mediante RPA se realizó con el programa PhotoScan ${ }^{\circledR}$ (Agisoft).

Para el tratamiento estadístico de los datos y el desarrollo de modelos numéricos se ha recurrido al programa de libre licencia $\mathrm{R}$ (R Core Team, 2015).

\section{Resultados}

El tratamiento de los ficheros de datos LiDAR ha permitido obtener la altura sobre el terreno de las diferentes cubiertas vegetales y su fracción de cabida cubierta, variables necesarias para la ulterior estimación de la biomasa a través de ecuaciones que ofrece la literatura científica. Además, a través del estudio de los índices de vegetación y las simulaciones con el modelo de crecimiento se consigue evaluar la situación de la zona IFM al comienzo del proyecto y su posible evolución en los siguientes años. 


\subsection{Modelos digitales.}

A partir del procesamiento de los datos LiDAR se obtienen los distintos MDTs mencionados anteriormente, como el modelo MDE mostrado en la Figura 6. Los vuelos realizados mediante RPA, por su parte, han hecho posible visualizar y analizar con gran detalle, las diferentes cubiertas vegetales para su identificación mediante ortofotos (ver Figura 7 izquierda). Estas imágenes son el resultado de la fusión de las fotografías individuales tomadas por el sensor como respuesta a la necesidad de tener en una sola imagen toda la zona de interés (Medina, Joyanes \& Pinilla, 2013).

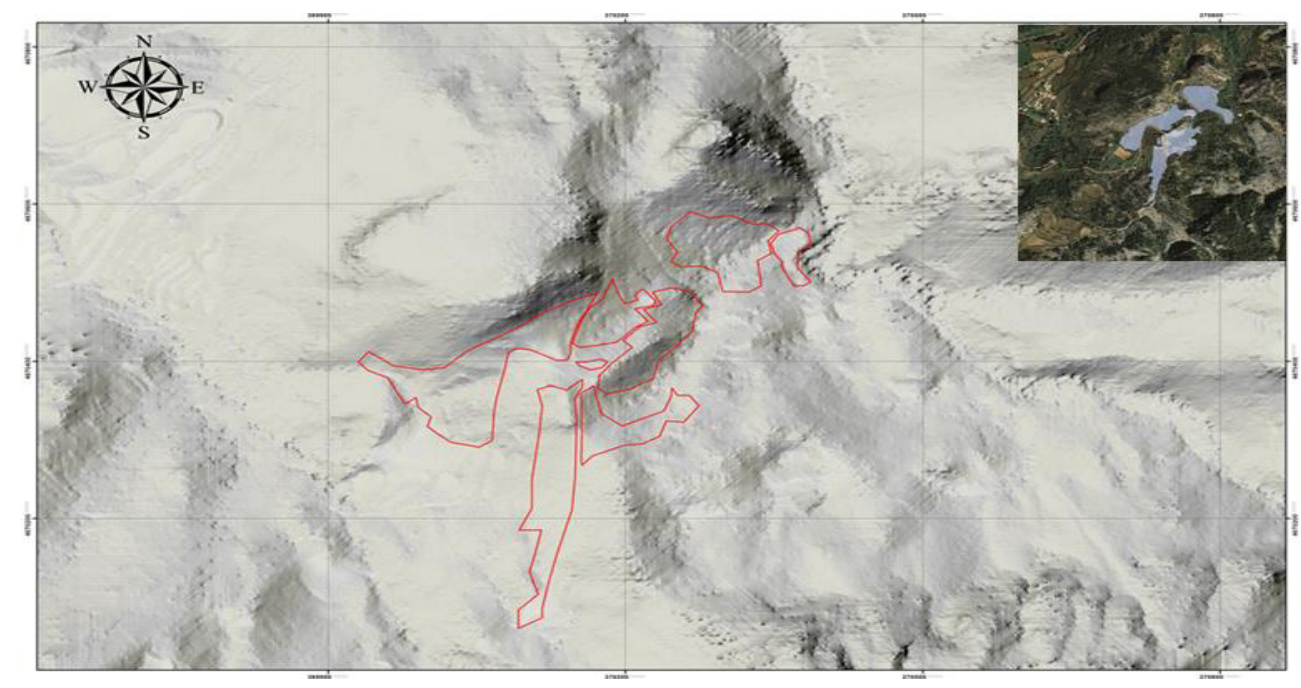

Figura 6 - MDE de algunas de las zonas ARR obtenido mediante procesamiento de datos LiDAR

El procesado de los vuelos con RPA permite también obtener modelos MDS de cada zona mediante software fotogramétrico (ver Figura 7 derecha).
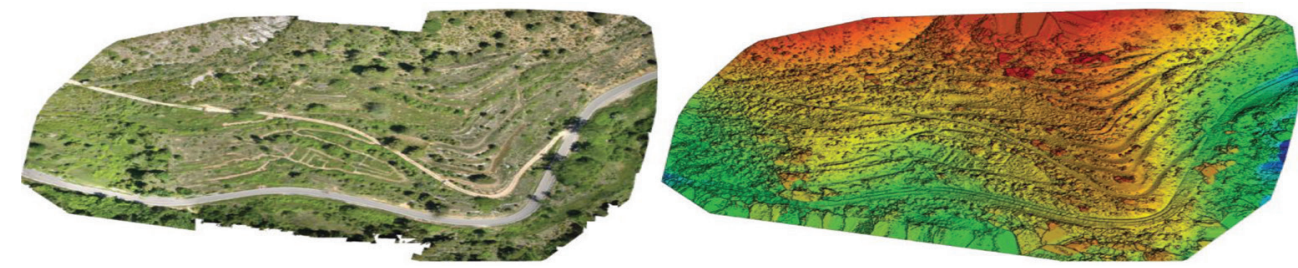

Figura 7 - Izquierda: ortofoto de una zona ARR generada a partir de la fusión de fotografías tomadas desde RPA. Derecha: MDS de la misma zona ARR generado mediante fotogrametría

\subsection{Estratificación}

El proceso de estratificación de la vegetación, a partir de los cálculos estadísticos realizados sobre los datos LiDAR y el apoyo de las ortofotos generadas mediante vuelo 
con RPA, es fundamental para poder aplicar posteriormente los modelos o ecuaciones de biomasa. Los resultados de dichos cálculos estadísticos se vuelcan en un SIG para cuantificar la superficie que ocupa cada estrato definido, en este caso: suelo, herbáceo, matorral y arbolado (ver Figura 8).

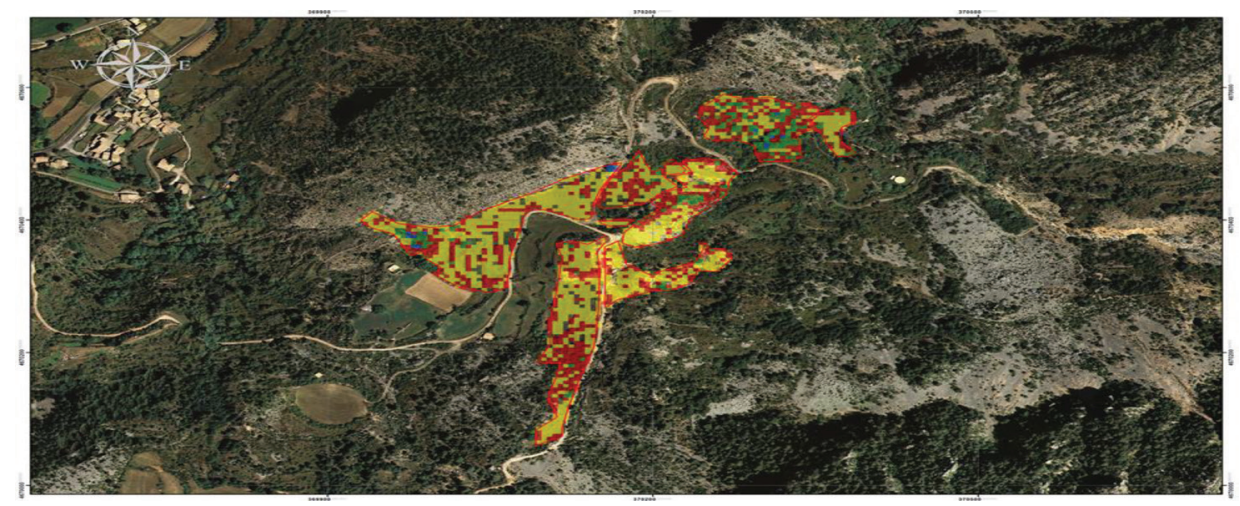

Figura 8 - Estratificación de vegetación a partir de datos LiDAR

A continuación se muestran los resultados (Tabla 1) para la estratificación de las zonas ARR.

\begin{tabular}{llllllllll}
\hline $\begin{array}{l}\text { Zona } \\
\text { ARR }\end{array}$ & $\begin{array}{l}\text { Superficie } \\
\text { total }\end{array}$ & Suelo & & \multicolumn{2}{c}{ FCC herbáceo } & \multicolumn{2}{c}{ FCC matorral } & \multicolumn{2}{c}{ FCC arbolado } \\
\hline & $\mathbf{m}^{\mathbf{2}}$ & $\mathbf{m}^{\mathbf{2}}$ & $\mathbf{\%}$ & $\mathbf{m}^{\mathbf{2}}$ & $\mathbf{\%}$ & $\mathbf{m}^{\mathbf{2}}$ & $\mathbf{\%}$ & $\mathbf{m}^{\mathbf{2}}$ & $\mathbf{\%}$ \\
\hline $\mathbf{1}$ & 5960 & 1644,95 & 27,60 & 1822,40 & 30,58 & 2391,67 & 40,13 & 100,99 & 1,69 \\
\hline $\mathbf{2}$ & 15948 & 7112,12 & 44,60 & 5859,54 & 36,74 & 2854,95 & 17,90 & 121,39 & 0,76 \\
\hline 3 & 3382 & 729,44 & 21,57 & 1199,95 & 35,48 & 1283,45 & 37,95 & 169,16 & 5,00 \\
\hline 4 & 350 & 57,90 & 16,54 & 259,41 & 74,12 & 32,69 & 9,34 & 0,00 & 0,00 \\
\hline 5 & 13373 & 5877,46 & 43,95 & 4592,45 & 34,34 & 2819,74 & 21,09 & 83,35 & 0,62 \\
\hline 7 & 1058 & 301,68 & 28,51 & 465,89 & 44,04 & 290,43 & 27,45 & 0,00 & 0,00 \\
\hline 8 & 3899 & 1009,87 & 25,90 & 1088,86 & 27,93 & 1691,20 & 43,38 & 109,07 & 2,80 \\
\hline 9 & 14467 & 3252,48 & 43,00 & 2744,80 & 36,29 & 1547,30 & 20,46 & 19,43 & 0,26 \\
\hline Total: & 66000 & 25626,49 & 38,83 & 21587,29 & 32,71 & 17628,86 & 26,71 & 1158,36 & 1,76 \\
\hline & & & & & & & & & \\
\hline
\end{tabular}

Tabla 1 - Superficie ocupada por cada estrato

\subsection{Individualización de arbolado}

A partir de los datos LIDAR se han individualizado los árboles presentes en las zonas de estudio mediante un algoritmo automático de ventana variable (Popescu et al., 2002) y 
se han agrupado los mismos en función de su diámetro normal en clases diamétricas de 5 $\mathrm{cm}$ de amplitud. Se han considerado como pies menores aquellos árboles cuyo diámetro normal está comprendido entre $2,5 \mathrm{~cm}$ y 7,5 cm. En la Figura 9 se puede observar los resultados obtenidos para zonas ARR mediante un mapa generado a tal efecto:

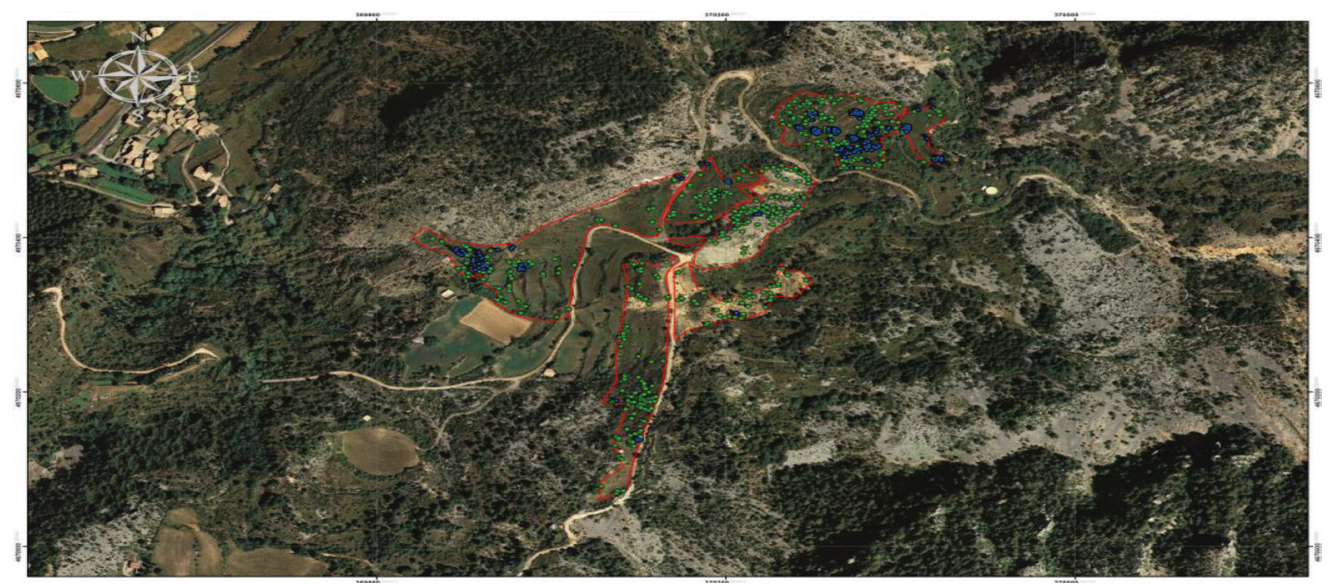

Figura 9 - Individualización de arbolado en zonas ARR: pies menores (verde) y pies mayores (azul)

\subsection{Modelos numéricos}

El proceso de procesamiento de datos LiDAR tiene como fase final la obtención de ecuaciones de regresión lineal donde se modela la relación entre una variable dependiente (biomasa, área basimétrica, número de pies...) y las variables independientes (estadísticos LiDAR). Se han examinado las relaciones de correlación entre las variables y se ha ido afinando los distintos modelos hasta conseguir altos coeficientes de determinación y bajos errores estándar (Tabla 2).

\begin{tabular}{|c|c|}
\hline Style Name & Format \\
\hline $\begin{array}{l}\text { Biomasa de la parcela = Elevación media }+ \text { Elevación percentil } \\
\text { o5 }+ \text { Elevación percentil } 50+\text { Elevación percentil } 75+\text { Elevación } \\
\text { Percentil } 90+\text { Elevación Percentil } 95+\text { FCC }+ \text { Elevación Percentil } \\
25+\text { Elevación CV + Elevación Percentil } 99\end{array}$ & $\begin{array}{l}\text { Residual standard error }=0,571 \\
\text { Multiple } R \text {-squared }=0,919 \\
\text { Ajusted } R \text {-squared }=0,853\end{array}$ \\
\hline $\begin{array}{l}\text { Área basimétrica = Elevación media + Elevación percentil o5 } \\
\text { + FCC + Elevación percentil a 50 + Elevación Percentil 95+ } \\
\text { Elevación Percentil 90 + Elevación CV + Elevación Percentil } 99 \text { + } \\
\text { Elevación variancia + Elevación stddev }\end{array}$ & $\begin{array}{l}\text { Residual standard error }=0,172 \\
\text { Multiple } R \text {-squared }=0,914 \\
\text { Ajusted } R \text {-squared }=0,843\end{array}$ \\
\hline
\end{tabular}

Tabla 2 - Modelos numéricos desarrollados a partir de estadísticos LiDAR

Cabe reseñar que al llevar estos modelos a un SIG para representar en un mapa (ver Figura 10) alguna variable, aparezcan celdas en blanco o "huecos" producto de pequeños errores que arroja el comando gridmetrics del programa FUSION que se traducen en celdas con información corrupta que han de ser desechadas. 


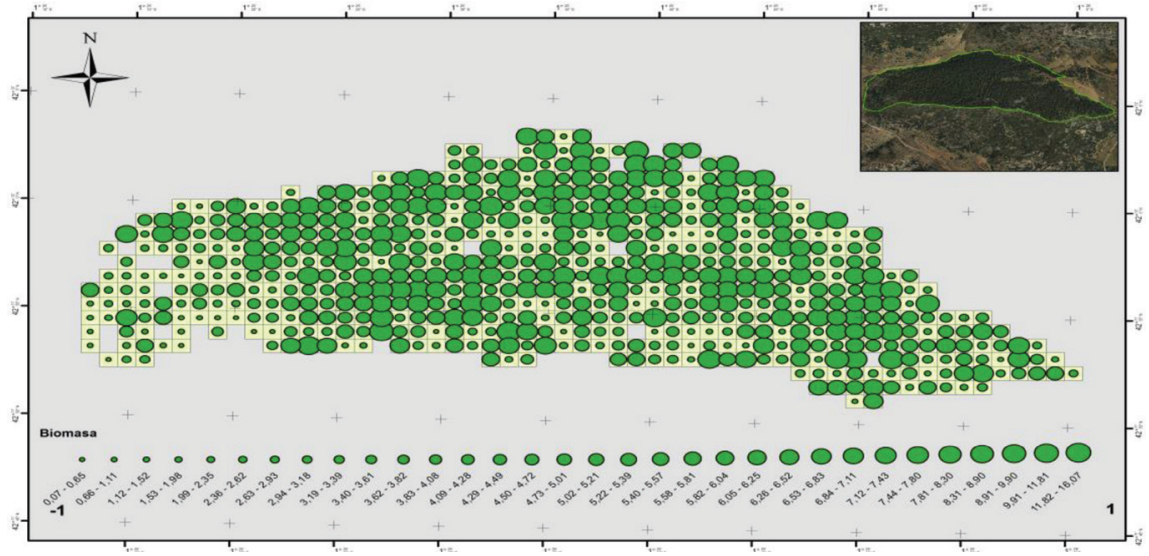

Figura 10 - Mapificación del modelo numérico de la variable biomasa

\section{5. Índices de vegetación.}

El análisis del índice NDVI (índice de Vegetación de Diferencia Normalizada), desde el año 1984 hasta el año 2011, calculado sobre imágenes multiespectrales del satélite Landsat 5, muestra claramente cómo el vigor de la masa ha disminuido (ver Figura 11), lo cual respalda el hecho de que se halla en un estado actual de estancamiento en su crecimiento debido a una alta densidad de arbolado.

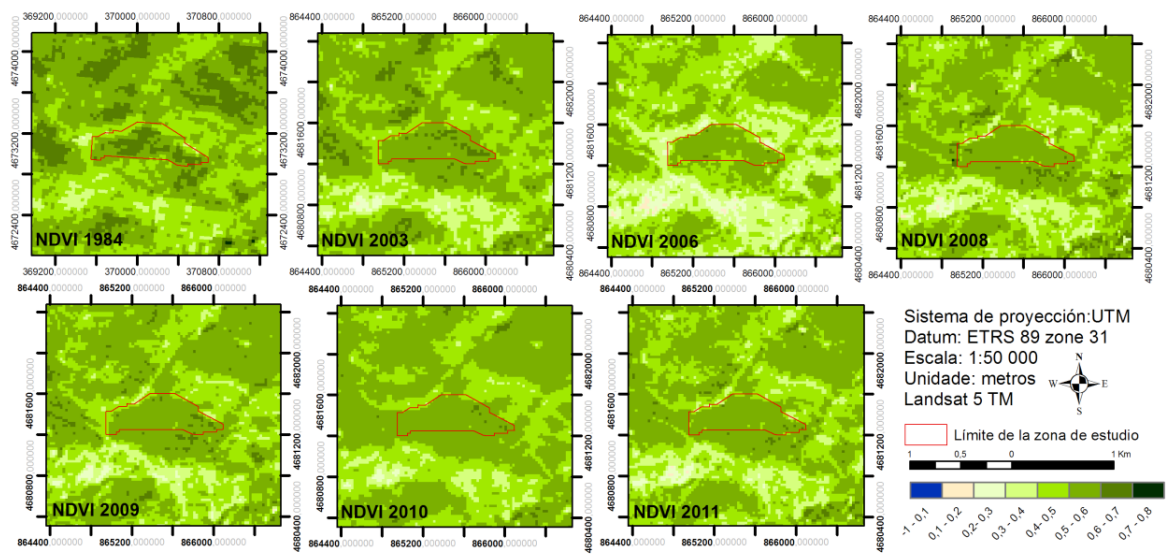

Figura 11 - Evolución del índice NDVI en la zona IFM

\subsection{Simulación.}

Las simulaciones realizadas con el software CO2FIX nos permiten concluir que con el modelo de gestión forestal Pso5, siempre que se incluyan los productos de las cortas como sumidero de carbono, la captura de $\mathrm{CO}_{2}$ es mayor que optando por no actuar sobre el bosque 
pues éste se halla prácticamente estancado en su crecimiento compitiendo por los recursos, además de presentarse mucho más susceptible a sufrir incendios, plagas y enfermedades.

En la Figura 12 izquierda se representa la evolución del carbono retenido en la biomasa aérea y radical de la masa forestal estudiada en el escenario en el que el bosque es sometido a los tratamientos silvícolas definidos por el modelo de gestión Pso5 con aclareos cada 10 años (se excluye en la representación el carbono retenido en los productos de la madera). Por otra parte, en la Figura 12 derecha se muestran tres de las simulaciones que se han realizado para representar la evolución del bosque en un escenario de no actuación, en el que se deja a la masa evolucionar de manera natural. Incluso bajo enfoques muy conservadores se infiere que la evolución natural, teniendo en cuenta las condiciones actuales del rodal, tiende a estancarse o incluso a disminuir la cantidad de carbono asimilado. Las distintas curvas responden a distintos grados de mortalidad, competencia y máximo de biomasa área en el rodal.
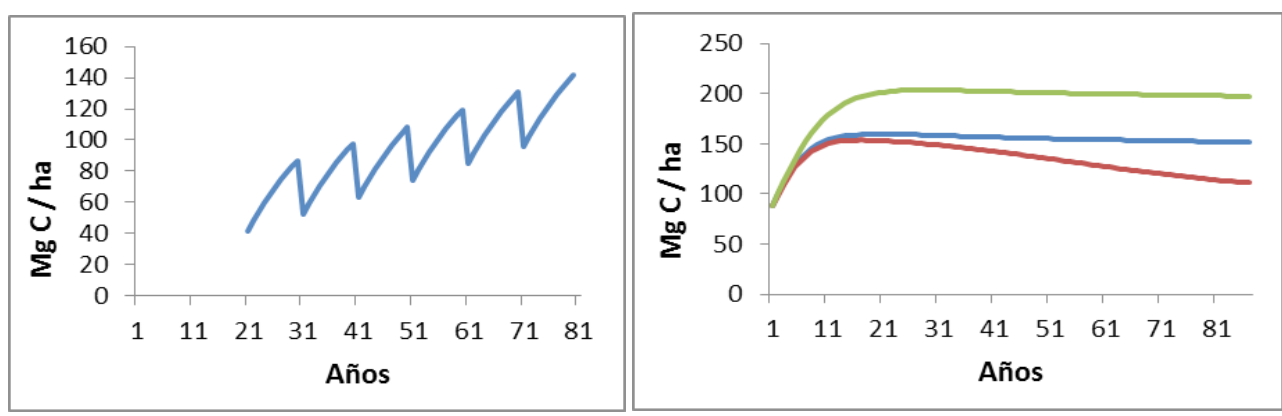

Figura 12 - Izquierda: evolución del carbono retenido en la biomasa de la zona IFM bajo gestión Pso5. Derecha: simulaciones de la evolución del carbono retenido por la biomasa en la zona IFM en escenarios de no actuación.

Estas simulaciones se han realizado adoptando criterios conservadores sin considerar riesgos propios de masas de alta densidad como incendios, plagas, enfermedades... y con la adopción de valores máximos de biomasa en el rodal altos, por lo que los resultados, en cuanto a carbono capturado, hubieran sido mucho más bajos para los escenarios sin intervención. Para ilustrarlo con un ejemplo: se conocen estudios de masas forestales similares, no gestionadas, de Pinus sylvestris en España, donde se ha monitorizado un crecimiento de 186 t/ha de biomasa aérea en 25 años (Ruiz-Peinado, Bravo-Oviedo, López-Senespleda, Río \& Montero, 2013). La masa de la zona IFM ha crecido únicamente 134,8 t/ha de biomasa aérea en 60 años. Para las simulaciones se ha utilizado un mínimo de 300 t/ha como máxima biomasa aérea a la que puede llegar el rodal en 80 años. Por lo tanto, la adopción de valores de máximo de biomasa aérea inferiores en el rodal (coherentes con los datos de crecimiento de la masa en los últimos años) conllevaría peores resultados para los casos en los que no se aplican los aclareos.

Paralelamente, la visualización de ortofotos históricas proporcionadas por el IGN (Figura 13), nos permite apreciar la evolución histórica del bosque de la zona IFM, el cual surgió con el abandono de las tierras de labor en los años 50 del siglo pasado y se ha ido desarrollando de manera espontánea, sin ser gestionado, hasta la situación de estancamiento al inicio del proyecto. 

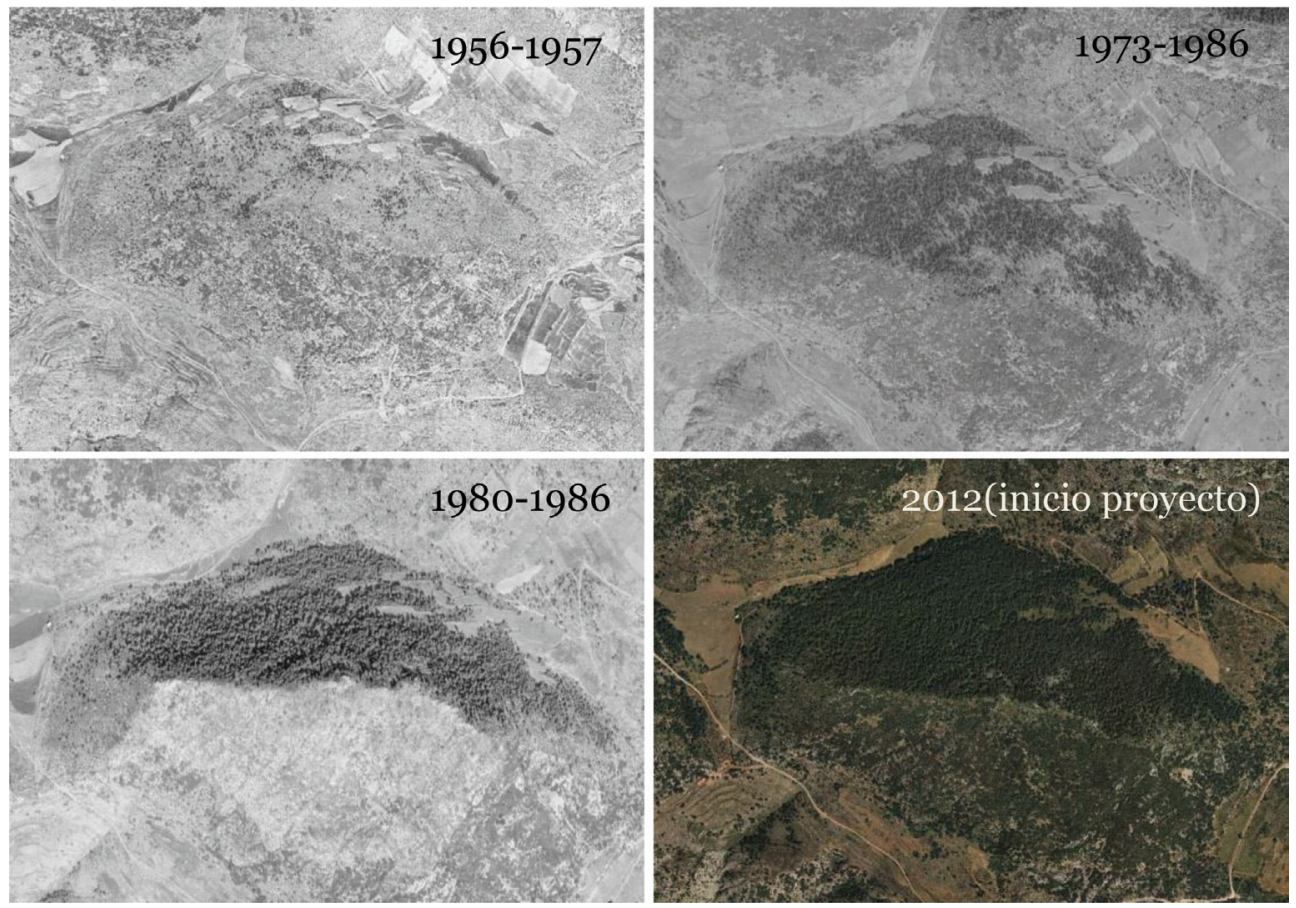

Figura 13 - Evolución de la zona IFM a través de ortofotos históricas.

\section{Conclusiones}

La combinación de tecnologías de teledetección utilizadas en este trabajo permite obtener información sobre las zonas de estudio en toda su extensión y no de manera puntual. De esta manera se reducen los errores por interpolación de datos muestrales o por poca representatividad de los mismos, habida cuenta de la heterogeneidad de las parcelas sobre las que se ha trabajado. Además, el uso de plataformas aéreas permite extraer fácilmente datos de zonas de otra manera inaccesibles, así como cubrir extensas áreas rápidamente. Esto supone un ahorro en tiempo, coste económico y en trabajo sobre el terreno, reducido a la toma de datos de apoyo y contraste.

Se ha demostrado, asimismo, que estas tecnologías son aptas para generar la información necesaria para la determinación de biomasa existente y, por lo tanto, de la cantidad de carbono retenido en la misma, permitiendo distinguir distintos estratos de vegetación. Estos resultados sirven, en el caso que nos ocupa, para cuantificar la línea base y establecer valores de referencia para evaluar la adicionalidad de las acciones del proyecto.

Además, a través del procesamiento de estadísticos LiDAR se han generado distintos modelos digitales del terreno así como modelos numéricos de diversos parámetros. Esta metodología ha sido complementada con éxito mediante fotogrametría de baja altura con RPA. 
Por otra parte, el estudio de los índices de vegetación, calculados sobre series históricas de imágenes multiespectrales de satélite, junto con las simulaciones ejecutadas en el modelo CO2FIX nos permiten determinar la idoneidad de la gestión forestal en el bosque de estudio, en cuanto a la asimilación de $\mathrm{CO}_{2}$ y paralelamente, a la prevención de enfermedades, plagas e incendios en comparación con un escenario de no intervención en dicho bosque.

Tanto la evolución de las plantaciones efectuadas en las zonas ARR, así como la incidencia de las actividades de gestión en la zona IFM concretadas en aclareos orientados a conseguir un incremento de biomasa que maximice la captura de $\mathrm{CO}_{2}$, podrán ser monitoreadas y evaluadas a través de las metodologías de teledetección utilizadas en este trabajo. De este modo se podrá cuantificar y contrastar la contribución del proyecto respecto a la situación de partida.

\section{Referencias bibliográficas.}

ArcGIS. Version 10.0. Redlands, CA: Environmental Systems Research Institute, Inc., 2010.

Baltsavias E. (2008). Introduction to airborne LIDAR and physical principles of LIDAR technology. Institute of Geodesy and Photogrammetry, ETH Zurich, Switzerland. $361 \mathrm{pp}$.

Croitoru, L., \& Merlo, M. (2005). Mediterranean forest values. In Valuing Mediterranean forests. Toward total economic value (pp. 378). L. Croitoru \& M. Merlo (Eds.), CABI Publishing.

García M., Riaño D., Chuvieco E. \& Danson F.M. (2010). Estimating biomass carbon stocks for a Mediterranean forest in central Spain using LiDAR height and intensity data. Remote Sensing of Environment, 114: 816-830.

Huete A. R. (1988). A soil-adjusted vegetation index (SAVI). Remote Sensing of Environment, 25(3): 295-309.

IPCC (2000). IPCC Special report. Land use, land use change and forestry. Summary for policy makers

Isenburg, M. (2008). LasTools-Efficient Tools for LiDAR Processing, Version 111216. http://lastools.org

Marklund, L. G., \& Schoene, D. (2006). Global assessment of growing stock, biomass and carbon stock. Global Forest Resources Assessment 2005. Rome: Forestry Department. Food and Agricultural Organization of the United Nations.

Medina, J., Joyanes., L. \& Pinilla, C. (2013). Aplicativo Web para la Fusión de Imágenes Satelitales. RISTI - Revista Ibérica de Sistemas y Tecnologías de Información, (11): 17-30. doi: 10.4304/risti.11.17-30

Moisés, J., Ibáñez, M., Rodríguez, R., Olarieta J.R. (2004). Estudi climatològic de la Vall d'Alinyà. In: Els sistemes naturals de la vall d'Alinyà. Barcelona: Institució Catalana d'Història Natural, p. 17-45. 
Montero, G., Ruiz-Peinado, R., \& Muñoz, M. (2005). Producción de biomasa y fijación de $\mathrm{CO}_{2}$ por los bosques españoles. Monografías INIA, Serie forestal $n^{o} 13,270 p$, Madrid.

Navarro-Cerrillo, R.M., Sánchez de la Orden M., Gómez Bonilla J., García Ferrer, A., Hernández Clemente, R., Lanjeri, S. (2010). Aplicación de imágenes LIDAR para la estimación del índice de superficie foliar (LAI) en encinas [Quercus ilex L. subsp. ballota (Desf.) Samp.]. Forests systems 19(1): 61-69.

Photoscan (2013). Agisoft LLC, San Petersburgo, Rusia.

Piqué, M., Beltrán, M., Vericat, P., Cervera, T., Farriol, R. \& Baiges, T. (2011) Models de gestió per als boscos de pi roig (Pinus sylvestris L.): producció de fusta i prevenció d'incendis forestals. Sèrie: Orientacions de gestió forestal sostenible per a Catalunya (ORGEST). Centre de la Propietat Forestal. Departament d'Agricultura, Ramaderia, Pesca, Alimentació i Medi Natural. Generalitat de Catalunya.

Popescu, S.C., Wynne, R.H., Nelson, R.F (2002). Estimating plot-level tree heights with lidar: local filtering with a canopy-height based variable window size. Computers and Electronics in Agriculture 37(1-3): 71-95.

Porta, J., Parapar, J., García, P., Fernández, G., Touriño, J., Ónega, F., ... Crecente, R. (2012). Sistema de Información del Banco de Tierras de Galicia. RISTI - Revista Ibérica de Sistemas e Tecnologias de Informação, (9): 27-41. doi: 10.4304/ risti.9.27-41.

QGIS Development Team (2015). QGIS Geographic Information System. Open Source Geospatial Foundation Project. http://qgis.osgeo.org

R Core Team (2013). R: A Language and Environment for Statistical Computing. $\mathrm{R}$ Foundation for Statistical Computing. Vienna, Austria. https://www.R-project.org

Renslow M., Geenfield P. \& Guay T. (2000). Evaluation of Multi-Return LIDAR for forestry applications. US Department of Agriculture Forest Service Engineering, Remote Sensing Applications. Recuperado en junio de 2016 de http://www.ndep. gov/USDAFS_LIDAR.pdf

Rouse, J. W.; Haas, R. H.; Schell, J. A.; Deering, D. W. 1979. Monitoring vegetation systems in the Great Plains with ERTS. Third ERTS Symposium, NASA SP-351 I, 309-317.

Ruiz-Peinado, R., Bravo-Oviedo, A., López-Senespleda, E., Río, M. \& Montero, G. (2013). ¿Cómo afectan las claras a las cantidades de carbono en una repoblación de Pinus sylvestris L.?. En actas del $6^{\circ}$ Congreso Forestal Español. Vitoria, España.

Sánchez Sastre, LF., Herrero Llorente, E., Hernández Navarro, S., Carrión Prieto, P., Garrido Lagunaga, F., Marcos Robles, J.L. \& Clérigo Pérez, Z. (2016). Proyecto LIFE+ Operación CO2: nuevas tecnologías aplicadas a la obtención de créditos de carbono mediante reforestación, restauración y gestión forestal. In Proceedings of the 6th Ibero-American Congress on Qualitative Research (CIAIQ2016), Qualitative Research on Engineering and Technology, vol 4. Porto, Portugal. 
Schelhaas, M.J, van Esch, P.W., Groen, T.A., de Jong, B.H.J., Kanninen, M., Liski, J. et al. (2004). CO2FIX V 3.1 - A modelling framework for quantifying carbon sequestration in forest ecosystems. Wageningen, Alterra, Alterra-rapport 1068.

Vázquez, M. Y. G., Sexto, C. F., Rocha, Á., \& Aguilera, A. (2016). Mobile Phones and Psychosocial Therapies with Vulnerable People: a First State of the Art. Journal of Medical Systems, 40(6): 1-12. 